\title{
Mini Review on the Impact of Mobile Parts' Exchange During the DAIR Procedure (Debridement, Antibiotics, Irrigation, Retention) for Infected Total Joint Arthroplasties
}

Pascal Bezel', Sandro F. Fucentese ${ }^{2}$, Jan Burkhard ${ }^{1,3}$, Dominique Holy ${ }^{1,3}$, Arend J. Nieuwland ${ }^{2}$, Marco Burkhard ${ }^{2}$, Ilker Uçkay ${ }^{1,2,3,4 *}$

'Internal Medicine, Balgrist University Hospital, Zurich, Switzerland

${ }^{2}$ Department of Orthopedic Surgery, Balgrist University Hospital, Zurich, Switzerland

${ }^{3}$ Infectiology, Balgrist University Hospital, Zurich, Switzerland

${ }^{4}$ Unit for Clinical and Applied Research, Balgrist University Hospital, Zurich, Switzerland

\section{Article Info}

\section{Article Notes}

Received: July 11, 2019

Accepted: August 12, 2019

\section{${ }^{*}$ Correspondence:}

Ilker Uçkay, MD, Balgrist University Hospitals, Forchstrasse 340, 8008 Zurich, Switzerland; Telephone No: +414438637 05; Fax No: +41 4438637 09; Email: Ilker.Uckay@balgrist.ch.

(c) 2019 Uckay I. This article is distributed under the terms of the Creative Commons Attribution 4.0 International License.

\section{Abstract}

A prosthetic joint infection (PJI) requires a combined approach (infectiology and surgery). The therapeutic DAIR approach (debridement, antibiotics, irrigation, and retention) is an option for acute and stable PJI yielding remission incidences that oscillate between $70 \%$ and $90 \%$; in a literature mostly composed of retrospective single-center trials. DAIR can be performed with or without mobile part's exchange during debridement. Scientific data proving the necessity of mobile part exchanges (by leaving other infected components in situ) remain scarce. In this narrative mini review, we evaluate the existing literature that analyses the benefit of exchanging mobile parts with at least ten own cases. We moreover discuss the optimal duration of concomitant targeted systemic antibiotic therapy and reveal some insights in the surgical difficulties in performing DAIR. Our conclusion tends to favor of the mobile part's exchange whenever feasible.

\section{Introduction}

A prosthetic joint infection (PJI) is a serious complication of arthroplasty surgery occurring in 0.8 to $1.9 \%$ of knee arthroplasties and 0.3 to $1.7 \%$ of hip arthroplasties ${ }^{1}$. PJIs require both antimicrobial treatment and surgical interventions. While the practice of the antibiotic therapy relies on uniform concepts throughout the world, the surgical approach resumes a higher variance and bases on the surgeon's experience or the co-morbidities of the patients. Possible strategies include a one- or two-stage implant exchange, resection arthroplasty (with or without arthrodesis), or DAIR ${ }^{1,2}$ (debridement, antibiotics, irrigation, and retention). Notably, DAIR can be used either curatively or as an initiation of a long-term suppressive antibiotic therapy ${ }^{3}$. The curative DAIR approach implies an acute infection with a stable implant and can reveal remissions up to 70\%$90 \%$ in selected patient population ${ }^{4,5}$. In this sense, the potential of DAIR lags behind the complete arthroplasty exchanges only by some percent ${ }^{6,7}$.

Scientific data proving the necessity of mobile part exchanges (polyethylene, liners, heads, inlays; by leaving other infected components in situ) remain scarce, although many experts advocate it for theoretical reasons ${ }^{1-9}$. In this narrative mini review, we evaluate existing literature regarding the benefit of exchanging mobile parts, discuss the optimal duration of concomitant targeted systemic 
antibiotic therapy, and reveal some insights in the surgical difficulties in performing DAIR. In contrast, we do not review the microbiology, the choice of antibiotic agents, the detailed surgical techniques, DAIR as suppressive purposes, or the demographics of DAIR patients around the world, for which specific literature are available.

\section{Methods}

We conducted a literature review that was oriented on the PRISMA procedure and targeted the benefits of mobile parts' exchange in DAIR of hip and knee PJIs; including all available publications with patient recruitment until 31 May 2019; with the MESH terms "DAIR", "infection" together with "exchange", "mobile parts" or "inlay" in English, German or French languages in PubMed and Google (Scholar). We also searched the reference lists of retrieved articles for additional papers. We excluded reviews, opinion papers, publications in other languages, publications without specific data on the mobile part's exchange, DAIR as suppressive therapy indications, DAIR in mycobacterial or fungal PJIs, and repeat papers issuing from the (presumed) same database (same author groups). For this mini literature review without providing own patient data, there were no ethical concerns. The primary outcome was "remission" linked to the presence or documented absence of previous mobile part's exchange.

\section{Results}

We retrieved twenty well documented articles reporting (more than) ten own DAIR cases, and that equally display information about mobile parts' exchanges ${ }^{5,8-27}$. All were retrospective analyses with a similar case-control design, but with different outcomes. In six studies, the exchange of the mobile parts was reported, but was not of important scientific interest, because we could not link exchange with remission outcomes. Fourteen studies explicitly investigated the prognostic value of the mobile parts' exchange and are now resumed in Table 1. Among them, six studies (resuming 1035 patients) reporting a benefit of the mobile part's exchange, and eight (605 cases) denied such a benefit (Table 1).

Overall, remission rates in DAIR with mobile parts' exchange ranged up to $90 \%$. Studies in favor of the exchange reported a two- or even four-fold increase in the benefit; probably independent of other co-factors. The described increase of the remission incidence of mobile parts' exchange ranged from 53\%-92\% (estimated interquartile range $65-85 \%$ ) compared to maximally $77 \%$ remission in a DAIR procedure explicitly without mobile parts' exchange (estimated interquartile range 60-70\%). In terms of infection remission, there seems to be no difference between the DAIR procedures in total hip or in total knee PJIs. The diversity of the articles, their contents, the detailed information displayed, and the patient population was too heterogonous to allow a statistically formal (pseudo)-meta-analysis.

Importantly, none of the studies reported an adverse event related to the mobile parts' exchange, but adverse events of the mobile part's exchange have never been the primary study objectives. One retrospective study looked for antibiotic-related adverse events in curative DAIR patients, and found no specific variations according the various substrata of the DAIR population ${ }^{18}$. Also, we lack information regarding other clinically important noninfectious outcomes (e.g. persistent pain or mechanical handicaps), surgical costs, and surgical difficulties when comparing between a DAIR for total hip or total knee PJIs ${ }^{1-}$ ${ }^{42}$. There is one exception: the functional outcomes of DAIR with a primary (uninfected) hip arthroplasty and a two-

Table 1: Mini-review on Infected Hip and/or Knee Total Joint Arthroplasties with the Emphasis on the Influence of Mobile-part's Changes During DAIR.

\begin{tabular}{|c|c|c|c|c|c|c|c|c|}
\hline First Author & Cases & $\begin{array}{c}\text { Mobile parts } \\
\text { exchanged }\end{array}$ & $\begin{array}{c}\text { Overall } \\
\text { Remission }\end{array}$ & $\begin{array}{c}\text { Reported benefit } \\
\text { of mobile parts' } \\
\text { exchange }\end{array}$ & $\begin{array}{l}\text { Remission with } \\
\text { mobile parts' } \\
\text { exchange }\end{array}$ & $\begin{array}{c}\text { Remission } \\
\text { without mobile } \\
\text { parts' exchange }\end{array}$ & Study design & Years \\
\hline Deirmengian ${ }^{24}$ & 31 & $10(32 \%)$ & $35 \%$ & none & $\mathrm{n} / \mathrm{a}$ & $\mathrm{n} / \mathrm{a}$ & retrospective & 1990-2000 \\
\hline Peel ${ }^{11}$ & 43 & $18(42 \%)$ & $86 \%$ & none & $83 \%$ (of 18) & $76 \%$ (of 25) & retrospective & $2000-2010$ \\
\hline Achermann ${ }^{5}$ & 50 & $26(52 \%)$ & $92 \%$ & none & $\mathrm{n} / \mathrm{a}$ & $\mathrm{n} / \mathrm{a}$ & retrospective & 2005-2010 \\
\hline Choi $^{21}$ & 28 & $19(68 \%)$ & $50 \%$ & none & $\mathrm{n} / \mathrm{a}$ & $\mathrm{n} / \mathrm{a}$ & retrospective & 1999-2007 \\
\hline Koh $^{14}$ & 52 & $49(94 \%)$ & $71 \%$ & none & $73 \%$ (of 49) & $33 \%$ (of 3 ) & retrospective & 2005-2012 \\
\hline Tornero $^{26}$ & 222 & $159(72 \%)$ & $77 \%$ & none & $77 \%$ (of 159) & $76 \%$ (of 63) & retrospective & 1999-2014 \\
\hline Bryen $^{35}$ & 112 & $63(56 \%)$ & $83 \%$ & none & $\mathrm{n} / \mathrm{a}$ & $\mathrm{n} / \mathrm{a}$ & retrospective & 2000-2012 \\
\hline Ottesen $^{12}$ & 67 & $18(31 \%)$ & $84 \%$ & none & $78 \%$ (of 18) & $90 \%$ (of 40$)$ & retrospective & 2008-2013 \\
\hline Lora-Tamayo $^{30}$ & 418 & $220(53 \%)$ & $58 \%$ & 2-fold benefit & $65 \%$ (of 211) & $48 \%$ (of 190) & retrospective & 2003-2012 \\
\hline Hirsiger $^{18}$ & 112 & $48(43 \%)$ & $84 \%$ & 2-fold benefit & $83 \%$ (of 48) & $84 \%$ (of 64 ) & retrospective & 2004-2017 \\
\hline Choi $^{20}$ & 32 & $19(59 \%)$ & $31 \%$ & 3-fold benefit & $53 \%$ (of 19) & $0 \%$ (of 13) & retrospective & 2002-2007 \\
\hline Wouthuyzen $^{8}$ & 323 & $176(54 \%)$ & $55 \%$ & 3-fold benefit & $63 \%$ (of 176 ) & $48 \%$ (of 147 ) & retrospective & 2005-2015 \\
\hline Grammatopoulos $^{19}$ & 122 & $65(53 \%)$ & $68 \%$ & 4-fold benefit & $92 \%$ (of 65) & $77 \%$ (of 57) & retrospective & 1997-2013 \\
\hline $\operatorname{Kim}^{15}$ & 28 & $11(39 \%)$ & $61 \%$ & 14-fold benefit & $91 \%$ (of 11 ) & $41 \%$ (of 17 ) & retrospective & 2003-2012 \\
\hline
\end{tabular}


stage arthroplasty exchange in hip PJI's were compared ${ }^{43}$. The DAIR group had an overall inferior functional Oxford Hip Score at five year's follow-up compared to the twostage exchange in hip PJI's (but similar remission of infection $)^{43}$ but the subpopulation that required only one DAIR debridement for eradication of infection had a mean score to the primary uninfected arthroplasty group.

\section{Discussion}

Motivated by our single-center analysis proving a benefit of the mobile part exchange in $\mathrm{DAIR}^{18}$, we add this minireview targeting to the statistical benefits of this exchange; which was always highly advocated by experts ${ }^{1-9}$, met its own review objectives, but was less underlined by solid scientific data. Indeed, so far the literature investigated showed other independent co-factors on the outcome of DAIR such as associations with pathogens ${ }^{30-41}$ (especially Staphylococcus aureus), choice of particular antimicrobial agents ${ }^{31}$, multidrug-resistance, polymicrobial PJI (especially involving fungi), bacteremic disease, the height of serum inflammation markers, presence of immune-suppression, active smoking, and subacute PJI's lasting since more than one month ${ }^{2,26-41}$.

Recent years acknowledged a statistical benefit in exchanging mobile parts, although this opinion is not shared by all research groups (Table 1). Hence, the statistical support for a widespread practice came later than the expert opinion ${ }^{1-9}$. However, and importantly, our "statistical support" bases on retrospective data with all possible biases and confounding by indications. As no data from randomized trials exist, we cannot exclude the hypothesis that perhaps more severely ill patients or with more complicated surgical issues were not submitted to exchange of mobile components. To give an extended example, the Grammatopoulos paper concludes that even in late PJI, exchange of mobile parts is better than their preservation during DAIR. However, it is not clear if exchange was performed more often in early that late PJI, in younger than older patients, or in patients who were pre-operated for $\mathrm{PJI}^{19,43}$. Other papers do not mention at all the reasons for this important choice regarding DAIR.

Furthermore, the exchange of mobile parts is always reported as a dichotomous parameter (exchange of mobile party conducted versus not conducted). Hence, details about the procedure (e.g. duration, methodical approach) are not taken into account. We also lack important clinical evaluations in the DAIR procedure (with and without exchange of mobile parts) such as functional outcomes, costs and quality of life assessments. The same is true for the surgical team and the course of the operation, which might play a role, however, cannot be measured by objective parameters and thus have not been included in any study. In order to preoperatively assess the risk of failure after DAIR, there have been efforts to develop scores like the
KLIC-Score for early acute PJI and the CRIME80-Score for acute hematogenous PJIs; both holding moderate evidence, but a strong international consensus ${ }^{2,8,26,29}$. The KLIC-Score has already been validated externally ${ }^{29}$.

Generally speaking, hematogenous total knee PJI's may occur later than their counterparts in the hip ${ }^{41}$, but we ignore if this is also true for the subpopulation of patients chosen for the curative DAIR approach. From an orthopedic surgeon's point of view, total knee arthroplasties in general may be associated with more post-surgical pain, reduced range of motion, and even stiffness than total hip arthroplasties ${ }^{9,14,19}$. Surgeons would also expect that knee DAIRs would be associated with more discomfort even after infection eradication, but specific data are lacking to the best of our knowledge. Technically speaking, there are no major differences in difficulty performing DAIR for knee compared to hip PJIs, even regarding the exchange of mobile parts; with the only exception of tibial insert ${ }^{16,23,36}$. Both articulations require the availability of individual exchange material, because every arthroplasty type and size has its own material produced by the manufacturer, which has to be replaced identically. To the best of our knowledge, we ignore if some author groups experienced DAIR with a replacement of mobile parts from another manufacturer. It seems not possible to insert a tibial inlay from different companies. In contrast, the change of the thickness of the tibial insert is possible; almost never into a smaller size, but frequently into a larger one to enhance the stability ${ }^{44}$.

The number of surgical debridements for one DAIR episode does not seem to enhance remission, i.e. a second look does not seem being more beneficiary than a single debridement with direct mobile parts exchange ${ }^{42}$. In Switzerland, the country of this mini review, the changing of inlays roughly costs 1000 US $\$$, a new prosthesis around 6000 \$, and a revision arthroplasty 8000 \$ or more according to the personal experience of the orthopedic surgeons in the last decade.

Although not the objective of this mini review, the duration of concomitant antibiotic therapy after DAIR is a subject of debate. Briefly, many centers treat either during six weeks, and many during twelve weeks or longer; independently of the involved pathogens, patient's comorbidities, or the exchange of mobile parts. According to available literature, the infection remission does not seem to differ between all these strategies, total antibiotic duration or modalities of antibiotic administrations (parenteral versus oral ${ }^{18,44,49,50}$. Other outcomes such a prosthetic stability or pain have not been investigated in relation with the duration of antibiotic therapy. A single-center evaluation specifically included the antibiotic duration in the final multivariate model and could not define a minimal duration of antibiotic prescription even when controlling 
to the exchange of mobile parts (while the exchange augmented the odds for remission by two-fold) ${ }^{18}$. Another multicenter retrospective study performed in Geneva and two other centers in France directly compared infection remission after curative DAIRs between exactly six weeks and twelve weeks of post-surgical antibiotic therapy and found no difference ${ }^{44}$. Other authors groups retrospectively found no benefit in prolonging systemic targeted antibiotic therapy beyond six weeks when the implant was left in place $^{45-48}$. As we could not find either retrospective or prospective data favoring a longer treatment than for six weeks, the authors of this mini review advocate a prospective trial regarding this topic, in as much as DAIR is gaining momentum in the world of PJI management.

\section{Conclusion}

Our literature review identified fourteen retrospective studies proclaiming (or denying) the benefit of mobile parts' exchange with approximately similar study designs and methods, while the latest and the biggest studies were rather in its favor. An international consensus on DAIR from February 2019 gives the following recommendation: 'Owing to the lack of conclusive evidence in the form of welldesigned prospective randomized trials and standardized protocols, only a moderate strength of recommendation is provided for exchanging the modular components during DAIR to reduce the PJI recurrence rate ${ }^{2}$.' On the other hand, the variability among definitions of remission vs. failure after treatment, the length of follow-up, different surgical techniques, and timing of DAIR after initial implantation, pathogens, antimicrobial resistance, different antibiotic protocols, different prosthesis designs, cannot be compared between studies. Logically, the choice for retention of mobile parts can be individualized, even if retrospective data demonstrates a more favorable outcome with exchange.

According to our own opinion, there are no medical downsides to the exchange of mobile parts as well as possible benefits from it. We thus routinely performed mobile parts' exchange whenever feasible within the first or second look, and without compromising the patient's being. For example, we would certainly not add another surgical intervention only just to comply with our own believes regarding a mobile part's exchange (in absence of other indications), when it had not been performed during the first debridement. A prospective, randomized trial able to close the debate is still needed, which would also imply the question on the duration of post-surgical antibiotic therapy.

\section{References}

1. Del Pozo JL, Patel R. Clinical practice. Infection associated with prosthetic joints. N Engl J Med. 2009; 361(8): 787-94.

2. Argenson JN, Arndt M, Babis G, et al. Hip and Knee Section, Treatment,
Debridement and Retention of Implant: Proceedings of International Consensus on Orthopedic Infections. J Arthroplasty. 2019; 34(2S): 399-419.

3. Rao N, Crossett LS, Sinha RK, et al. Long-term suppression of infection in total joint arthroplasty. Clin Orthop Relat Res. 2003; 414: 55-60.

4. Bradbury T, Fehring TK, Taunton M, et al. The fate of acute methicillinresistant Staphylococcus aureus periprosthetic knee infections treated by open debridement and retention of components. J Arthroplasty. 2009; 24(6 Suppl): 101-4.

5. Achermann Y, Stasch P, Preiss S, et al. Characteristics and treatment outcomes of 69 cases with early prosthetic joint infections of the hip and knee. Infection. 2014; 42: 511-9.

6. Kunutsor SK, Beswick AD, Whitehouse MR, et al. Debridement, antibiotics and implant retention for periprosthetic joint infections: A systematic review and meta-analysis of treatment outcomes. J Infect. 2018; 77(6): 479-88.

7. Haasper C, Buttaro M, Hozack W, et al. Irrigation and debridement. J Orthop Res. 2014; 32 (Suppl 1): 130-5.

8. Wouthuyzen-Bakker M, Sebillotte M, Lomas J, et al. Clinical outcome and risk factors for failure in late acute prosthetic joint infections treated with debridement and implant retention. J Infect. 2019; 78(1): 40-7.

9. Mont MA, Waldman B, Banerjee $\mathrm{C}$, et al. Multiple irrigation, debridement, and retention of components in infected total knee arthroplasty. J Arthroplasty. 1997; 12(4): 426-33.

10. Lora-Tamayo J, Senneville É, Ribera A, et al. The Not-So-Good Prognosis of Streptococcal Periprosthetic Joint Infection Managed by Implant Retention: The Results of a Large Multicenter Study. Clin Infect Dis. 2017; 64(12): 1742-52.

11. Peel TN, Buising KL, Dowsey MM, et al. Outcome of debridement and retention in prosthetic joint infections by methicillin-resistant staphylococci, with special reference to rifampin and fusidic acid combination therapy. Antimicrob Agents Chemother. 2013; 57(1): 350-5.

12. Ottesen CS, Troelsen A, Sandholdt H, et al. Acceptable Success Rate in Patients With Periprosthetic Knee Joint Infection Treated With Debridement, Antibiotics, and Implant Retention. J Arthroplasty. 2019; 34(2): 365-8.

13. Huotari K, Vuorinen M, Rantasalo M. High Cure Rate for Acute Streptococcal Prosthetic Joint Infections Treated With Debridement, Antimicrobials, and Implant Retention in a Specialized Tertiary Care Center. Clin Infect Dis. 2018; 67(8): 1288-90.

14. Koh IJ, Han SB, In Y, et al. Open debridement and prosthesis retention is a viable treatment option for acute periprosthetic joint infection after total knee arthroplasty. Arch Orthop Trauma Surg. 2015; 135(6): 847-55.

15. Kim JG, Bae JH, Lee SY, et al. The parameters affecting the success of irrigation and debridement with component retention in the treatment of acutely infected total knee arthroplasty. Clin Orthop Surg. 2015; 7(1): 69-76.

16. Geurts JAP, Janssen DMC, Kessels AGH, et al. Good results in postoperative and hematogenous deep infections of 89 stable total hip and knee replacements with retention of prosthesis and local antibiotics. Acta Orthop. 2013; 84(6): 509-16.

17. Duque AF, Post ZD, Lutz RW, et al. Is There Still a Role for Irrigation and Debridement With Liner Exchange in Acute Periprosthetic Total Knee Infection? J Arthroplasty. 2017; 32(4): 1280-4.

18. Hirsiger S, Betz M, Stafylakis D, et al. The Benefice of Mobile Parts> Exchange in the Management of Infected Total Joint Arthroplasties with Prosthesis Retention (DAIR Procedure). J Clin Med. 2019; 8(2) doi.org/10.3390/jcm8020226. 
19. Grammatopoulos G, Kendrick B, McNally M, et al. Outcome Following Debridement, Antibiotics, and Implant Retention in Hip Periprosthetic Joint Infection-An 18-Year Experience. J Arthroplasty. 2017; 32(7): 2248-55.

20. Choi HR, von Knoch F, Zurakowski D, et al. Can implant retention be recommended for treatment of infected TKA? Clin Orthop Relat Res. 2011; 469(4): 961-9.

21. Choi HR, von Knoch F, Kandil AO, et al. Retention treatment after periprosthetic total hip arthroplasty infection. Int Orthop. 2012; 36(4): 723-9.

22. Buller LT, Sabry FY, Easton RW, et al. The preoperative prediction of success following irrigation and debridement with polyethylene exchange for hip and knee prosthetic joint infections. J Arthroplasty. 2012; 27(6): 857-64.

23. Bryan AJ, Abdel MP, Sanders TL, et al. Irrigation and Debridement with Component Retention for Acute Infection After Hip Arthroplasty: Improved Results with Contemporary Management. J Bone Joint Surg Am. 2017; 99(23): 2011-8.

24. Deirmengian C, Greenbaum J, Lotke PA, et al. Limited success with open debridement and retention of components in the treatment of acute Staphylococcus aureus infections after total knee arthroplasty. J Arthroplasty. 2003; 18: 22-6.

25. Konigsberg BS, Della Valle CJ, Ting NT, et al. Acute hematogenous infection following total hip and knee arthroplasty. J Arthroplasty. 2014; 29: 469-72.

26. Tornero E, Morata L, Martínez-Pastor JC, et al. KLIC-score for predicting early failure in prosthetic joint infections treated with debridement, implant retention and antibiotics. Clin Microbiol Infect. 2015; 21(8): 9-17.

27. Tsang STJ, Ting J, Simpson AHRW, et al. Outcomes following debridement, antibiotics and implant retention in the management of periprosthetic infections of the hip: a review of cohort studies. Bone Joint J. 2017; 99-B(11): 1458-66.

28. Sidhu MS, Cooper G, Jenkins N, et al. Prosthetic fungal infections: poor prognosis with bacterial co-infection. Bone Joint J. 2019; 101-B(5): 582-8.

29. Löwik CAM, Jutte PC, Tornero E, et al. Predicting Failure in Early Acute Prosthetic Joint Infection Treated With Debridement, Antibiotics, and Implant Retention: External Validation of the KLIC Score. J Arthroplasty. 2018; 33(8): 2582-7.

30. Lora-Tamayo J, Murillo O, Iribarren JA, et al. A large multicenter study of methicillin-susceptible and methicillin-resistant Staphylococcus aureus prosthetic joint infections managed with implant retention. Clin Infect Dis. 2013; 56(2): 182-94.

31. Berdal JE, Skråmm I, Mowinckel P, et al. Use of rifampicin and ciprofloxacin combination therapy after surgical debridement in the treatment of early manifestation prosthetic joint infections. Clin Microbiol Infect. 2005; 11(10): 843-5.

32. Holmberg A, Thórhallsdóttir VG, Robertsson 0, et al. 75\% success rate after open debridement, exchange of tibial insert, and antibiotics in knee prosthetic joint infections. Acta Orthop. 2015; 86(4): 457-62.

33. Aboltins CA, Page MA, Buising KL, et al. Treatment of staphylococcal prosthetic joint infections with debridement, prosthesis retention and oral rifampicin and fusidic acid. Clin Microbiol Infect. 2007; 13(6): 586-91.

34. Jaén N, Martínez-Pastor JC, Muñoz-Mahamud E, et al. Long-term outcome of acute prosthetic joint infections due to gram-negative bacilli treated with retention of prosthesis. Rev Esp Quimioter. 2012; 25(3): 194-8.
35. Byren I, Bejon P, Atkins BL, et al. One hundred and twelve infected arthroplasties treated with (DAIR) (debridement, antibiotics and implant retention): antibiotic duration and outcome. J Antimicrob Chemother. 2009; 63(6): 1264-71.

36. Laffer RR, Graber P, Ochsner PE, et al. Outcome of prosthetic kneeassociated infection: evaluation of 40 consecutive episodes at a single centre. Clin Microbiol Infect. 2006; 12(5): 433-9.

37. Martínez-Pastor JC, Muñoz-Mahamud E, Vilchez F, et al. Outcome of acute prosthetic joint infections due to gram-negative bacilli treated with open debridement and retention of the prosthesis. Antimicrob Agents Chemother. 2009; 53(11): 4772-7.

38. Vilchez F, Martínez-Pastor JC, García-Ramiro S, et al. Outcome and predictors of treatment failure in early post-surgical prosthetic joint infections due to Staphylococcus aureus treated with debridement. Clin Microbiol Infect. 2011; 17: 439-44.

39. Westberg M, Grøgaard B, Snorrason F. Early prosthetic joint infections treated with debridement and implant retention: 38 primary hip arthroplasties prospectively recorded and followed for median 4 years. Acta Orthop. 2012; 83: 227-32.

40. Puhto AP, Puhto T, Syrjala H. Short-course antibiotics for prosthetic joint infections treated with prosthesis retention. Clin Microbiol Infect. 2012; 18(11): 1143-8.

41. Vu DL, Uçkay I, Gonzalez A, et al. Factors related to outcome of early and delayed prosthetic joint infections. J Infect. 2016; 72(2): 255-7.

42. Kuiper JWP, Vos SJ, Rachid Saouti R, et al. Prosthetic joint-associated infections treated with DAIR (debridement, antibiotics, irrigation, and retention): Analysis of risk factors and local antibiotic carriers in 91 patients. Acta Orthop. 2013; 84(4): 380-6.

43. Grammatopoulos G, Bolduc ME, Atkins BL, et al. Functional outcome of debridement, antibiotics and implant retention in periprosthetic joint infection involving the hip: a case-control study. Bone Joint J. 2017; 99-B(5): 614-22.

44. Chaussade H, Uçkay I, Vuagnat A, et al. Antibiotic therapy duration for prosthetic joint infections treated by Debridement and Implant Retention (DAIR): Similar long-term remission for 6 weeks as compared to 12 weeks. Int J Infect Dis. 2017; 63: 37-42.

45. Bernard L, Legout L, Zürcher-Pfund L, et al. Six weeks of antibiotic treatment is sufficient following surgery for septic arthroplasty. J Infect. 2010; 61(2): 125-32.

46. Farhad R, Roger PM, Albert C, et al. Six weeks antibiotic therapy for all bone infections: results of a cohort study. Eur J Clin Microbiol Infect Dis. 2010; 29(2): 217-22.

47. Yen HT, Hsieh RW, Huang CY, et al. Short-course versus long-course antibiotics in prosthetic joint infections: a systematic review and meta-analysis of one randomized controlled trial plus nine observational studies. J Antimicrob Chemother. 2019; doi: 10.1093/ jac/dkz166.

48. Al-Mayahi M, Betz M, Müller DA, et al. Remission rate of implantrelated infections following revision surgery after fractures. Int Orthop. 2013; 37(11): 2253-8.

49. Zürcher-Pfund L, Uçkay I, Legout L, et al. Pathogen-driven decision for implant retention in the management of infected total knee prostheses. Int Orthop. 2013; 37(8): 1471-5.

50. Betz M, Abrassart S, Vaudaux P, et al. Increased risk of joint failure in hip prostheses infected with Staphylococcus aureus treated with debridement, antibiotics and implant retention compared to Streptococcus. Int Orthop. 2015; 39(3): 397-401. 